\title{
Norm and Essential Norm of Composition Followed by Differentiation from Logarithmic Bloch Spaces to $H_{\mu}^{\infty}$
}

\author{
Shanli Ye \\ Department of Mathematics, Fujian Normal University, Fuzhou 350007, China \\ Correspondence should be addressed to Shanli Ye; shanliye@fjnu.edu.cn \\ Received 25 February 2014; Revised 25 March 2014; Accepted 28 March 2014; Published 14 May 2014 \\ Academic Editor: Ljubisa Kocinac \\ Copyright (c) 2014 Shanli Ye. This is an open access article distributed under the Creative Commons Attribution License, which \\ permits unrestricted use, distribution, and reproduction in any medium, provided the original work is properly cited. \\ In this note we express the norm of composition followed by differentiation $D C_{\varphi}$ from the logarithmic Bloch and the little \\ logarithmic Bloch spaces to the weighted space $H_{\mu}^{\infty}$ on the unit disk and give an upper and a lower bound for the essential norm \\ of this operator from the logarithmic Bloch space to $H_{\mu}^{\infty}$.
}

\section{Introduction}

Let $\mathbb{D}=\{z:|z|<1\}$ be the open unit disk in the complex plane $\mathbb{C}, H(\mathbb{D})$ be the space of all analytic functions on $\mathbb{D}$, and $H^{\infty}$ be the space of bounded analytic functions on $\mathbb{D}$ with the norm $\|f\|_{\infty}=\sup _{z \in \mathbb{D}}|f(z)|$.

An analytic function $f \in H(\mathbb{D})$ is said to belong to the logarithmic Bloch space $\mathscr{L} \mathscr{B}$ if

$$
\|f\|_{\mathscr{L} \mathscr{B}}=\sup \left\{(1-|z|) \ln \left(\frac{2 e}{1-|z|}\right)\left|f^{\prime}(z)\right|: z \in \mathbb{D}\right\}<\infty
$$

and to the little logarithmic Bloch space $\mathscr{L} \mathscr{B}_{0}$ if

$$
\lim _{|z| \rightarrow 1^{-}}(1-|z|) \ln \left(\frac{2 e}{1-|z|}\right)\left|f^{\prime}(z)\right|=0 .
$$

It can be easily proved that $\mathscr{L} \mathscr{B}$ is a Banach space, under the norm

$$
\|f\|_{\mathscr{L}}=|f(0)|+\|f\|_{\mathscr{L} \mathscr{B}},
$$

and that $\mathscr{L} \mathscr{B} 0$ is a closed subspace of $\mathscr{L} \mathscr{B}$. Some sources for results and references about the logarithmic Bloch functions are the papers of Yoneda [1], Stević [2], and the authors of [3-8].
Let $\mu$ be a weight, that is, a positive continuous function on $\mathbb{D}$. The weighted space $H_{\mu}^{\infty}$ consists of all $f \in H(\mathbb{D})$ such that

$$
\|f\|_{H_{\mu}^{\infty}}=\sup _{z \in \mathbb{D}} \mu(z)|f(z)|<\infty
$$

where $\mu$ is a weight.

Let $\varphi$ be a holomorphic self-map of $\mathbb{D}$. The composition operator $C_{\varphi}$ is defined by

$$
C_{\varphi}(f)(z)=f(\varphi(z)), \quad f \in H(\mathbb{D}) .
$$

Let $D$ be the differentiation operator. The product $D C_{\varphi}$ is defined by

$$
D C_{\varphi}(f)=(f \circ \varphi)^{\prime}=f^{\prime}(\varphi) \varphi^{\prime}, \quad f \in H(\mathbb{D}) .
$$

The operator $D C_{\varphi}$ is probably studied for the first time by Hibschweiler and Portnoy in [9], where the boundedness and compactness of $D C_{\varphi}$ between Bergman and Hardy spaces are investigated. In [10], Stević calculated the norm of the operator $D C_{\varphi}$ from the classical Bloch space to $H_{\mu}^{\infty}$. Recently there has been some interest in calculating operator norms and essential norms of composition and related operators (see, e.g., [11-18] and the references therein). Motivated by the papers $[10,19]$, we continue here this line of research by calculating $\left\|D C_{\varphi}\right\|_{\mathscr{L} \mathscr{B} \rightarrow H_{\mu}^{\infty}}$. 
Suppose that $X_{1}$ and $X_{2}$ are Banach spaces and $L$ : $X_{1} \rightarrow X_{2}$ is a bounded linear operator. The essential norm $\|L\|_{e, X_{1} \rightarrow X_{2}}$ of $L$ is its distance to the compact operators. More precisely,

$$
\|L\|_{e, X_{1} \rightarrow X_{2}}=\inf \left\{\|L-K\|_{X_{1} \rightarrow X_{2}}:\right.
$$

$K$ a compact operator of $X_{1}$ into $\left.X_{2}\right\}$,

where $\|\cdot\|_{X_{1} \rightarrow X_{2}}$ denotes the operator norm. If $X_{1}=X_{2}$, it is simply denoted by $\|\cdot\|_{e}$. Since the set of all compact operators is a closed subset of the set of bounded operators, it follows that an operator $L$ is compact if and only if $\|L\|_{e, X_{1} \rightarrow X_{2}}=0$.

Essential norm formulas for composition operators are known in various settings. When $C_{\varphi}$ acts from the Hardy space $H^{2}(\mathbb{D})$ to itself, Shapiro [20] gives a formula for $\left\|C_{\varphi}\right\|_{e}$ in terms of the Nevanlinna counting function for $\varphi$. In [21], Donaway gives upper and lower estimates for $\left\|C_{\varphi}\right\|_{e}$ when $C_{\varphi}$ maps the Bloch, Dirichlet, or a Besov type space to itself. The essential norm of the $D C_{\varphi}$ operator from $\alpha$-Bloch spaces to $H_{\mu}^{\infty}$ space was estimated recently by Stević in [10]. In this note we give upper and lower estimates for $\left\|D C_{\varphi}\right\|_{e, \mathscr{L} \mathscr{B} \rightarrow H_{\mu}^{\infty}}$.

\section{The Operator Norm of $D C_{\varphi}: \mathscr{L} \mathscr{B}$ (or

$$
\left.\mathscr{L} \mathscr{B}_{0}\right) \rightarrow H_{\mu}^{\infty}
$$

In this section we prove a nice formula. Namely, we calculate the norm of the operator $D C_{\varphi}: \mathscr{L} \mathscr{B}\left(\right.$ or $\left.\mathscr{L} \mathscr{B}_{0}\right) \rightarrow H_{\mu}^{\infty}$.

Theorem 1. Assume $\mu$ is a weight on $\mathbb{D}$. Then the following are equivalent:

(a) $D_{\varphi}: \mathscr{L} \mathscr{B} \rightarrow H_{\mu}^{\infty}$ is a bounded operator;

(b) $D C_{\varphi}: \mathscr{L} \mathscr{B}_{0} \rightarrow H_{\mu}^{\infty}$ is a bounded operator;

(c) $\sup _{z \in \mathbb{D}}\left(\mu(z)\left|\varphi^{\prime}(z)\right|\right) /((1-|\varphi(z)|) \ln (2 e /(1-|\varphi(z)|)))<$ $\infty$

\section{Moreover, one has}

$$
\begin{aligned}
\left\|D C_{\varphi}\right\|_{\mathscr{L}_{\mathscr{B}_{0} \rightarrow H_{\mu}^{\infty}}} & =\left\|D C_{\varphi}\right\|_{\mathscr{L} \mathscr{B} \rightarrow H_{\mu}^{\infty}} \\
& =\sup _{z \in \mathbb{D}} \frac{\mu(z)\left|\varphi^{\prime}(z)\right|}{(1-|\varphi(z)|) \ln (2 e /(1-|\varphi(z)|))} .
\end{aligned}
$$

Proof. $(a) \Rightarrow(b)$. By the fact $\mathscr{L} \mathscr{B}_{0} \subset \mathscr{L} \mathscr{B}$ and the definition of operator norm, we easily obtain that $D C_{\varphi}: \mathscr{L}_{\mathscr{B}_{0}} \rightarrow H_{\mu}^{\infty}$ is a bounded operator and

$$
\left\|D C_{\varphi}\right\|_{\mathscr{L} \mathscr{B}_{0} \rightarrow H_{\mu}^{\infty}} \leq\left\|D C_{\varphi}\right\|_{\mathscr{L} \mathscr{B} \rightarrow H_{\mu}^{\infty}} .
$$

$(b) \Rightarrow(c)$. Suppose that $D C_{\varphi}$ is a bounded operator from $\mathscr{L} \mathscr{B}_{0}$ to $H_{\mu}^{\infty}$. Taking the test function $f(z)=z \in \mathscr{L} \mathscr{B}_{0}$, we easily have

$$
\begin{aligned}
\mu(w)\left|\varphi^{\prime}(w)\right| & \leq\left\|\varphi^{\prime}\right\|_{H_{\mu}^{\infty}}=\left\|D C_{\varphi}(z)\right\|_{H_{\mu}^{\infty}} \\
& \leq\left\|D C_{\varphi}\right\|_{\mathscr{L} \mathscr{B}_{0} \rightarrow H_{\mu}^{\infty}}\|z\|_{\mathscr{L}} \\
& =\left\|D C_{\varphi}\right\|_{\mathscr{L} \mathscr{B}_{0} \rightarrow H_{\mu}^{\infty}} \ln 2 e,
\end{aligned}
$$

for every $w \in \mathbb{D}$. It implies that (c) holds when $\varphi(z)=0$.

Fixing $w \in \mathbb{D} \backslash\{0\}$, we consider the function

$$
f_{w}(z)=\frac{1}{\bar{w}} \ln \ln \left(\frac{2 e}{1-\bar{w} z}\right)-\frac{1}{\bar{w}} \ln \ln 2 e .
$$

Since $r(x)=x \ln (2 e / x)$ is increasing on $(0,2]$ and $f_{w}(0)=$ 0 , we have

$$
\begin{aligned}
\left\|f_{w}\right\|_{\mathscr{L}}= & \sup _{z \in \mathbb{D}}(1-|z|) \ln \left(\frac{2 e}{1-|z|}\right) \\
& \times \frac{1}{|\ln (2 e /(1-\bar{w} z))|} \frac{1}{|1-\bar{w} z|} \\
\leq & \sup _{z \in \mathbb{D}} \frac{(1-|z|) \ln (2 e /(1-|z|))}{(1-|\bar{w} z|) \ln (2 e /(1-|\bar{w} z|))} \\
& \times \frac{(1-|\bar{w} z|) \ln (2 e /(1-|\bar{w} z|))}{|1-\bar{w} z| \ln (2 e /(1-\bar{w} z))} \leq 1 .
\end{aligned}
$$

Moreover, since

$$
\begin{aligned}
& (1-|z|) \ln \frac{2 e}{1-|z|}\left|f_{w}^{\prime}(z)\right| \\
& \leq \frac{(1-|z|) \ln (2 e /(1-|z|))}{(1-|\bar{w} z|) \ln (2 e /(1-|\bar{w} z|))} \\
& \quad \leq \frac{(1-|z|) \ln (2 e /(1-|z|))}{(1-|w|) \ln 2 e} \longrightarrow 0,
\end{aligned}
$$

as $|z| \rightarrow 1^{-}$, it follows that $f_{w} \in \mathscr{L} \mathscr{B}_{0}$ for every $w \in \mathbb{D} \backslash\{0\}$. Thus, for each $t \in(0,1)$ we obtain that

$$
\begin{aligned}
\left\|D C_{\varphi}\right\|_{\mathscr{L} \mathscr{B}_{0} \rightarrow H_{\mu}^{\infty}} & \geq\left\|D C_{\varphi}\left(f_{t(\varphi(w) /|\varphi(w)|)}\right)\right\|_{H_{\mu}^{\infty}} \\
& =\sup _{z \in \mathbb{D}} \mu(z)\left|\varphi^{\prime}(z) f_{t(\varphi(w) /|\varphi(w)|)}^{\prime}(\varphi(z))\right| \\
& \geq \frac{\mu(w)\left|\varphi^{\prime}(w)\right|}{(1-t|\varphi(w)|) \ln (2 e /(1-t|\varphi(w)|))},
\end{aligned}
$$

for every $\varphi(w) \neq 0$. Letting $t \rightarrow 1^{-}$, we obtain that

$$
\left\|D C_{\varphi}\right\|_{\mathscr{L}_{\mathscr{B}_{0} \rightarrow H_{\mu}^{\infty}}} \geq \frac{\mu(w)\left|\varphi^{\prime}(w)\right|}{(1-|\varphi(w)|) \ln (2 e /(1-|\varphi(w)|))},
$$

for every $\varphi(w) \neq 0$. It implies that (c) also holds when $\varphi(z) \neq 0$. 
$(c) \Rightarrow(a)$. For every $f \in \mathscr{L} \mathscr{B}$, we easily obtain that

$$
\begin{aligned}
\left\|D C_{\varphi} f\right\|_{H_{\mu}^{\infty}} & \leq \sup _{z \in D} \mu(z)\left|\left(D C_{\varphi} f\right)(z)\right| \\
& =\sup _{z \in D} \mu(z)\left|\varphi^{\prime}(z) f^{\prime}(\varphi(z))\right| \\
& \leq \sup _{z \in D} \frac{\mu(z)\left|\varphi^{\prime}(z)\right|}{(1-|\varphi(z)|) \ln (2 e /(1-|\varphi(z)|))}\|f\|_{\mathscr{L}} .
\end{aligned}
$$

Hence $D C_{\varphi}: \mathscr{L} \mathscr{B} \rightarrow H_{\mu}^{\infty}$ is a bounded operator. Also, we obtain

$$
\left\|D C_{\varphi}\right\|_{\mathscr{L} \mathscr{B} \rightarrow H_{\mu}^{\infty}} \leq \sup _{z \in \mathbb{D}} \frac{\mu(z)\left|\varphi^{\prime}(z)\right|}{(1-|\varphi(z)|) \ln (2 e /(1-|\varphi(z)|))}
$$

Moreover, from (9), (10), (15), and (17), we obtain

$$
\begin{aligned}
\left\|D C_{\varphi}\right\|_{\mathscr{L}_{\mathscr{B}_{0} \rightarrow H_{\mu}^{\infty}}} & =\left\|D C_{\varphi}\right\|_{\mathscr{L} \mathscr{B} \rightarrow H_{\mu}^{\infty}} \\
& =\sup _{z \in \mathbb{D}} \frac{\mu(z)\left|\varphi^{\prime}(z)\right|}{(1-|\varphi(z)|) \ln (2 e /(1-|\varphi(z)|))} .
\end{aligned}
$$

\section{Estimates of Essential Norm of}

$$
D C_{\varphi}: \mathscr{L} \mathscr{B}\left(\text { or } \mathscr{L} \mathscr{B}_{0}\right) \rightarrow H_{\mu}^{\infty}
$$

In this section we will estimate the essential norm of $D C_{\varphi}$ : $\mathscr{L} \mathscr{B}\left(\right.$ or $\left.\mathscr{L} \mathscr{B}_{0}\right) \rightarrow H_{\mu}^{\infty}$. For this purpose we need some lemmas.

Lemma 2. If $f \in \mathscr{L} \mathscr{B}$, then $|f(z)| \leq(1 / 2+\ln \ln (e /(1-$ $|z|)))\|f\|_{\mathscr{L}}$.

This can be done in exactly the same way as in the proof of [3, Lemma 2.1].

Lemma 3. Let $\varphi$ be an analytic self-map of $\mathbb{D}$ and $\mu$ be a weight on $\mathbb{D}$. Assume that $\mathrm{DC}_{\varphi}$ is a bounded operator from $\mathscr{L} \mathscr{B}\left(\right.$ or $\left.\mathscr{L} \mathscr{B}_{0}\right)$ to $H_{\mu}^{\infty}$; then $D C_{\varphi}$ is compact if and only if for any bounded sequence $\left\{f_{n}\right\}$ in $\mathscr{L} \mathscr{B}$ (or $\left.\mathscr{L} \mathscr{B}_{0}\right)$, which converges to 0 uniformly on compact subsets of $\mathbb{D}$, one has $\left\|D C_{\varphi}\left(f_{n}\right)\right\|_{H_{\mu}^{\infty}} \rightarrow 0$ as $n \rightarrow \infty$.

Proof. Necessity. Suppose that $D C_{\varphi}: \mathscr{L} \mathscr{B}\left(\right.$ or $\left.\mathscr{L} \mathscr{B}_{0}\right) \rightarrow H_{\mu}^{\infty}$ is compact. Let $\left\{f_{n}\right\}$ be a bounded sequence in $\mathscr{L} \mathscr{B}\left(\right.$ or $\left.\mathscr{L} \mathscr{B}_{0}\right)$ with $f_{n} \rightarrow 0$ uniformly on compact subsets of $D$. Assume that there is a subsequence $\left\{f_{n_{k}}\right\}$ and an $\epsilon_{0}>0$ such that $\left\|D C_{\varphi} f_{n_{k}}\right\| \geq \epsilon_{0}$ for all $k=1,2,3, \ldots$. Since $D C_{\varphi}$ is compact, we can find a further subsequence $\left\{f_{n_{k_{j}}}\right\}$ and a function $f \in H_{\mu}^{\infty}$ such that $\lim _{j \rightarrow \infty}\left\|D C_{\varphi} f_{n_{k_{j}}}-f\right\|_{H_{\mu}^{\infty}}=0$. Then we obtain that, for $z \in D$,

$$
\left|\left(D C_{\varphi} f_{n_{k_{j}}}-f\right)(z)\right| \leq \frac{\left\|D C_{\varphi} f_{n_{k_{j}}}-f\right\|_{H_{\mu}^{\infty}}}{\mu(z)} .
$$

Hence $D C_{\varphi} f_{n_{k_{j}}}-f \rightarrow 0$ uniformly on compact subsets of $D$. Also, since $f_{n_{k_{j}}} \rightarrow 0$ uniformly on compact subsets of $D$, $D C_{\varphi} f_{n_{k_{j}}} \rightarrow 0$ uniformly on compact subsets of $D$. It follows that $f=0$ and hence $\lim _{j \rightarrow \infty}\left\|D C_{\varphi} f_{n_{k_{j}}}\right\|_{H_{\mu}^{\infty}}=0$, contradicting the fact that $\left\|D C_{\varphi} f_{n_{k}}\right\| \geq \epsilon_{0}$ for all $k=1,2,3, \ldots$ Therefore we must have that $\lim _{n \rightarrow \infty}\left\|D C_{\varphi}\left(f_{n}\right)\right\|_{H_{\mu}^{\infty}}=0$.

Sufficiency. Let $\left\{f_{n}\right\}$ be a bounded sequence in $\mathscr{L} \mathscr{B}$ (or $\left.\mathscr{L} \mathscr{B}_{0}\right)$. Then Lemma 2 and Montel's Theorem tell us that $\left\{f_{n}\right\}$ forms a normal family, and hence there exists a subsequence $\left\{f_{n_{k}}\right\}$ converging uniformly on compact sets to some function $f$. It is easy to see that $f$ must be in $\mathscr{L} \mathscr{B}\left(\mathscr{L} \mathscr{B}_{0}\right)$. Then $\left\{f_{n_{k}}-f\right\}$ is a bounded sequence in $\mathscr{L} \mathscr{B}\left(\right.$ or $\mathscr{L} \mathscr{B} \mathscr{B}_{0}$ ) converging to 0 uniformly on compact subsets of $\mathbb{D}$ and by the hypothesis guarantees that $D C_{\varphi} f_{n_{k}} \rightarrow D C_{\varphi} f$ in $H_{\mu}^{\infty}$. Thus $D C_{\varphi}$ is compact.

Lemma 4. Let $\mu$ be a weight on $\mathbb{D}$ and $\varphi$ be an analytic selfmap of $\mathbb{D}$ with $\|\varphi\|_{\infty}<1$. Suppose that $D C_{\varphi}: \mathscr{L} \mathscr{B}$ (or $\left.\mathscr{L} \mathscr{B}_{0}\right) \rightarrow H_{\mu}^{\infty}$ is bounded. Then $\mathrm{DC}_{\varphi}: \mathscr{L} \mathscr{B}\left(\right.$ or $\left.\mathscr{L} \mathscr{B}_{0}\right) \rightarrow$ $H_{\mu}^{\infty}$ is compact.

Proof. Suppose that $\left\{f_{n}\right\}$ is a bounded sequence in $\mathscr{L} \mathscr{B}\left(\right.$ or $\mathscr{L} \mathscr{B}_{0}$ ) which converges to 0 uniformly on compact subsets of $\mathbb{D}$. By Cauchy's inequality we easily obtain that $\left\{f_{n}^{\prime}\right\}$ also converges to 0 uniformly on compact subsets of $\mathbb{D}$. Since $D C_{\varphi}$ is bounded, one can take the test function $f(z)=z$ to see that $\varphi^{\prime} \in H_{\mu}^{\infty}$. Then we obtain that

$$
\left\|D C_{\varphi} f_{n}\right\|_{H_{\mu}^{\infty}} \leq\left\|\varphi^{\prime}\right\|_{H_{\mu}^{\infty}} \sup _{w \in \varphi(\mathbb{D})}\left|f_{n}^{\prime}(w)\right| \longrightarrow 0
$$

as $n \rightarrow \infty$, since $\varphi(\mathbb{D})$ is contained in the disk $|w| \leq\|\varphi\|_{\infty}<$ 1 , which is a compact subset of $\mathbb{D}$. Hence, by Lemma 3 , the operator $D C_{\varphi}: \mathscr{L} \mathscr{B}\left(\right.$ or $\left.\mathscr{L} \mathscr{B}_{0}\right) \rightarrow H_{\mu}^{\infty}$ is compact.

Lemma 5. Let $f \in \mathscr{L} \mathscr{B}$. Then $\left\|f_{t}\right\|_{\mathscr{L}} \leq\|f\|_{\mathscr{L}}, 0<t<1$, where $f_{t}(z)=f(t z)$.

Since $r(x)=(1-x) \ln (2 e /(1-x))$ is decreasing on $[0,1)$, one may easily prove the result. 
Theorem 6. Let $\mu$ be a weight on $\mathbb{D}$ and $\varphi$ be an analytic selfmap of $\mathbb{D}$. Suppose that $D C_{\varphi}: \mathscr{L} \mathscr{B}\left(\right.$ or $\left.\mathscr{L} \mathscr{B}_{0}\right) \rightarrow H_{\mu}^{\infty}$ is bounded. Then

$$
\begin{aligned}
& \frac{1}{2} \limsup _{|\varphi(z)| \rightarrow 1^{-}} \frac{\mu(z)\left|\varphi^{\prime}(z)\right|}{(1-|\varphi(z)|) \ln (2 e /(1-|\varphi(z)|))} \\
& \leq\left\|D C_{\varphi}\right\|_{e, \mathscr{L} \mathscr{B}_{0} \rightarrow H_{\mu}^{\infty}} \leq\left\|D C_{\varphi}\right\|_{e, \mathscr{L} \mathscr{B} \rightarrow H_{\mu}^{\infty}} \\
& \leq 2 \limsup _{|\varphi(z)| \rightarrow 1^{-}} \frac{\mu(z)\left|\varphi^{\prime}(z)\right|}{(1-|\varphi(z)|) \ln (2 e /(1-|\varphi(z)|))} .
\end{aligned}
$$

Proof. If $\|\varphi\|_{\infty}<1$, by Lemma 4, it follows that $D C_{\varphi}$ : $\mathscr{L} \mathscr{B}\left(\right.$ or $\left.\mathscr{L} \mathscr{B}_{0}\right) \rightarrow H_{\mu}^{\infty}$ is compact which is equivalent to $\left\|D C_{\varphi}\right\|_{e, \mathscr{L} \mathscr{B}_{0} \rightarrow H_{\mu}^{\infty}}=\left\|D C_{\varphi}\right\|_{e, \mathscr{L} \mathscr{B} \rightarrow H_{\mu}^{\infty}}=0$. On the other hand, it is clear that in this case the condition $|\varphi(z)| \rightarrow 1$ is vacuous, so that it is understood that

$$
\limsup _{|\varphi(z)| \rightarrow 1^{-}} \frac{\mu(z)\left|\varphi^{\prime}(z)\right|}{(1-|\varphi(z)|) \ln (2 e /(1-|\varphi(z)|))}=0 .
$$

Now suppose that $\|\varphi\|_{\infty}=1$. Assume that $\left\{z_{n}\right\}$ is a sequence in $\mathbb{D}$ such that $\left|\varphi\left(z_{n}\right)\right| \rightarrow 1$ as $n \rightarrow \infty$. Let

$$
\begin{aligned}
f_{n}(z)= & \frac{1}{2 \overline{\varphi\left(z_{n}\right)} a_{n}}\left(\ln \ln \frac{2 e}{1-\overline{\varphi\left(z_{n}\right) z}}\right)^{2} \\
& -\frac{1}{\overline{2\left(z_{n}\right)} a_{n}}(\ln \ln 2 e)^{2}
\end{aligned}
$$

where $a_{n}=\ln \ln \left(2 e /\left(1-\left|\varphi\left(z_{n}\right)\right|^{2}\right)\right)$. Then we have $f_{n}(0)=0$,

$$
f_{n}^{\prime}\left(\varphi\left(z_{n}\right)\right)=\frac{1}{\left(1-\left|\varphi\left(z_{n}\right)\right|^{2}\right) \ln \left(2 e /\left(1-\left|\varphi\left(z_{n}\right)\right|^{2}\right)\right)} .
$$

Clearly $f_{n}(z) \rightarrow 0$ uniformly on compact subsets of $\mathbb{D}$ as $n \rightarrow \infty$. It follows that

$$
\begin{aligned}
\left\|f_{n}\right\|_{\mathscr{L}}= & \sup _{z \in \mathbb{D}}(1-|z|) \ln \frac{2 e}{1-|z|} \frac{1}{a_{n}}\left|\ln \ln \frac{2 e}{1-\overline{\varphi\left(z_{n}\right)} z}\right| \\
& \times \frac{1}{\left|\ln \left(2 e /\left(1-\overline{\varphi\left(z_{n}\right)} z\right)\right)\right|} \overline{\left|1-\overline{\varphi\left(z_{n}\right)} z\right|} \\
\leq & \sup _{z \in \mathbb{D}} \frac{2 \pi+\ln \left(2 \pi+\ln \left(2 e /\left(1-\left|\varphi\left(z_{n}\right)\right|\right)\right)\right)}{\ln \ln \left(2 e /\left(1-\mid \varphi\left(\left.z_{n}\right|^{2}\right)\right)\right.} \\
& \times \frac{(1-|z|) \ln (2 e /(1-|z|))}{\left(1-\left|\overline{\varphi\left(z_{n}\right)} z\right|\right) \ln \left(2 e /\left(1-\left|\overline{\varphi\left(z_{n}\right)} z\right|\right)\right)} \\
& \times \frac{\left(1-\left|\overline{\varphi\left(z_{n}\right)} z\right|\right) \ln \left(2 e /\left(1-\left|\overline{\varphi\left(z_{n}\right)} z\right|\right)\right)}{\left|1-\overline{\varphi\left(z_{n}\right)} z\right| \ln \left(2 e /\left|1-\overline{\varphi\left(z_{n}\right)} z\right|\right)} \\
\leq & \frac{2 \pi+\ln \left(2 \pi+\ln \left(2 e /\left(1-\left|\varphi\left(z_{n}\right)\right|\right)\right)\right)}{\ln \ln \left(2 e /\left(1-\left|\varphi\left(z_{n}\right)\right|^{2}\right)\right)}
\end{aligned}
$$

Thus, $\lim \sup _{n \rightarrow \infty}\left\|f_{n}\right\|_{\mathscr{L}} \leq 1$. Let $g_{n}=f_{n} /\left\|f_{n}\right\|_{\mathscr{L}}$. Then $\left\|g_{n}\right\|_{\mathscr{L}}=1$ and $g_{n} \rightarrow 0$ uniformly on compact subsets of $\mathbb{D}$ as $n \rightarrow \infty$. Since $g_{n} \in \mathscr{L} \mathscr{B}_{0}$, then it follows that $g_{n}$ converges to 0 weakly in $\mathscr{L} \mathscr{B}_{0}$. Thus, for any compact operator $K$ : $\mathscr{L} \mathscr{B}_{0} \rightarrow H_{\mu}^{\infty}, \lim _{n \rightarrow \infty}\left\|K g_{n}\right\|_{H_{\mu}^{\infty}}=0$. Therefore

$$
\begin{aligned}
\left\|D C_{\varphi}-K\right\|_{\mathscr{L}_{\mathscr{B}_{0}} \rightarrow H_{\mu}^{\infty}} & =\sup _{\|f\|_{\mathscr{L}} \leq 1}\left\|\left(D C_{\varphi}-K\right) f\right\|_{H_{\mu}^{\infty}} \\
& \geq \limsup _{n \rightarrow \infty}\left\|\left(D C_{\varphi}-K\right) g_{n}\right\|_{H_{\mu}^{\infty}} \\
& \geq \limsup _{n \rightarrow \infty}\left\|D C_{\varphi} g_{n}\right\|_{H_{\mu}^{\infty}} .
\end{aligned}
$$

Hence

$$
\begin{aligned}
\| D C_{\varphi} & \|_{e, \mathscr{L}_{0} \rightarrow H_{\mu}^{\infty}} \\
& \geq \limsup _{n \rightarrow \infty}\left\|D C_{\varphi} g_{n}\right\|_{H_{\mu}^{\infty}} \\
& =\limsup _{n \rightarrow \infty} \sup _{z \in \mathbb{D}}\left|\mu(z) g_{n}^{\prime}(\varphi(z)) \varphi(z)\right| \\
& \geq \limsup _{n \rightarrow \infty} \frac{1}{\left\|f_{n}\right\|_{\mathscr{L}}}\left|\mu\left(z_{n}\right) f_{n}^{\prime}\left(\varphi\left(z_{n}\right)\right) \varphi\left(z_{n}\right)\right| \\
& \geq \limsup _{n \rightarrow \infty} \frac{\mu\left(z_{n}\right)\left|\varphi\left(z_{n}\right)\right|}{\left(1-\left|\varphi\left(z_{n}\right)\right|^{2}\right) \ln \left(2 e /\left(1-\left|\varphi\left(z_{n}\right)\right|^{2}\right)\right)} \\
& =\frac{1}{2} \limsup _{n \rightarrow \infty} \frac{\mu\left(z_{n}\right)\left|\varphi\left(z_{n}\right)\right|}{\left(1-\left|\varphi\left(z_{n}\right)\right|\right) \ln \left(2 e /\left(1-\left|\varphi\left(z_{n}\right)\right|\right)\right)} .
\end{aligned}
$$

Thus the first inequality in (21) follows. The second inequality in (21) is obvious. Now we prove the third one.

Let $s \in(0,1)$ be fixed and $\rho_{n}=1-1 /(n+1), n=1,2, \ldots$ By Lemma 4 we obtain that the operator $D C_{\rho_{n} \varphi}: \mathscr{L} \mathscr{B} \rightarrow H_{\mu}^{\infty}$ is compact for every $n$. It follows that

$$
\begin{aligned}
\left\|D C_{\varphi}\right\|_{e, \mathscr{L} \mathscr{B} \rightarrow H_{\mu}^{\infty}} \leq & \left\|D C_{\varphi}-D C_{\rho_{n} \varphi}\right\|_{\mathscr{L} \mathscr{B} \rightarrow H_{\mu}^{\infty}} \\
= & \sup _{\|f\|_{\mathscr{L}} \leq 1}\left\|\left(D C_{\varphi}-D C_{\rho_{n} \varphi}\right)(f)\right\|_{H_{\mu}^{\infty}} \\
= & \sup _{\|f\|_{\mathscr{L}} \leq 1} \sup _{|\varphi(z)| \leq s} \mu(z)\left|\varphi^{\prime}(z)\right| \\
& \times\left|f^{\prime}(\varphi(z))-\rho_{n} f^{\prime}\left(\rho_{n} \varphi(z)\right)\right| \\
& +\sup _{\|f\|_{\mathscr{L}} \leq 1|\varphi(z)|>s} \sup (z)\left|\varphi^{\prime}(z)\right| \\
& \times\left|f^{\prime}(\varphi(z))-\rho_{n} f^{\prime}\left(\rho_{n} \varphi(z)\right)\right| \triangleq I_{1}+I_{2} .
\end{aligned}
$$


By Cauchy's inequality, we obtain that

$$
\begin{aligned}
& I_{1} \leq \sup _{\|f\|_{\mathscr{L}} \leq 1} \sup _{|\varphi(z)| \leq s}\left\|\varphi^{\prime}\right\|_{H_{\mu}^{\infty}}\left|f^{\prime}(\varphi(z))-f^{\prime}\left(\rho_{n} \varphi(z)\right)\right| \\
& +\sup _{\|f\|_{\mathscr{L}} \leq 1} \sup _{|\varphi(z)| \leq s}\left\|\varphi^{\prime}\right\|_{H_{\mu}^{\infty}}\left(1-\rho_{n}\right)\left|f^{\prime}\left(\rho_{n} \varphi(z)\right)\right| \\
& \leq\left(1-\rho_{n}\right)\left\|\varphi^{\prime}\right\|_{H_{\mu}^{\infty}} \sup _{\|f\|_{\mathscr{L}} \leq 1} \sup _{|w| \leq s}\left|f^{\prime \prime}(w)\right| \\
& +\left(1-\rho_{n}\right)\left\|\varphi^{\prime}\right\|_{H_{\mu}^{\infty}} \sup _{\|f\|_{\mathscr{L}} \leq 1} \sup _{|w| \leq s}\left|f^{\prime}(w)\right| \\
& \leq\left(1-\rho_{n}\right)\left\|\varphi^{\prime}\right\|_{H_{\mu}^{\infty}} \sup _{\|f\|_{\mathscr{L}} \leq 1} \frac{2}{1-s} \max _{|z| \leq(1+s) / 2}\left|f^{\prime}(z)\right| \\
& +\left(1-\rho_{n}\right)\left\|\varphi^{\prime}\right\|_{H_{\mu}^{\infty}} \sup _{\|f\|_{\mathscr{L}} \leq 1} \sup _{|w| \leq s}\left|f^{\prime}(w)\right|
\end{aligned}
$$

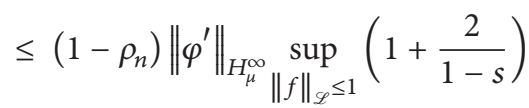

$$
\begin{aligned}
& \times \max _{|z| \leq(1+s) / 2} \frac{(1-|z|) \ln (2 e /(1-|z|))\left|f^{\prime}(z)\right|}{(1-|z|) \ln (2 e /(1-|z|))} \\
& \leq \frac{1}{n+1}\left\|\varphi^{\prime}\right\|_{H_{\mu}^{\infty}}\left(1+\frac{2}{1-s}\right) \frac{2}{(1-s) \ln (4 e /(1-s))} .
\end{aligned}
$$

On the other hand, by Lemma 5, we obtain that

$$
\begin{aligned}
& I_{2} \leq \sup _{\|f\|_{\mathscr{L}} \leq 1} \sup _{|\varphi(z)|>s} \frac{\mu(z)\left|\varphi^{\prime}(z)\right|\|f\|_{\mathscr{L}}}{(1-|\varphi(z)|) \ln (2 e /(1-|\varphi(z)|))} \\
&+\sup _{\|f\|_{\mathscr{L}^{\leq}} \leq 1} \sup _{|\varphi(z)|>s} \frac{\mu(z)\left|\varphi^{\prime}(z)\right|\left\|f_{\rho_{n}}\right\|_{\mathscr{L}}}{\left(1-\rho_{n}|\varphi(z)|\right) \ln \left(2 e /\left(1-\rho_{n}|\varphi(z)|\right)\right)} \\
& \leq \quad \sup _{\|f\|_{\mathscr{L}} \leq 1} \sup _{|\varphi(z)|>s} \frac{\mu(z)\left|\varphi^{\prime}(z)\right|\|f\|_{\mathscr{L}}}{(1-|\varphi(z)|) \ln (2 e /(1-|\varphi(z)|))} \\
& \leq 2 \sup _{|\varphi(z)|>s} \frac{\mu(z)\left|\varphi^{\prime}(z)\right|}{(1-|\varphi(z)|) \ln (2 e /(1-|\varphi(z)|))},
\end{aligned}
$$

where $f_{\rho_{n}}(z)=f\left(\rho_{n} z\right)$. Hence, for for all $s \in(0,1)$ and all $n$, we have

$$
\begin{aligned}
\left\|D C_{\varphi}\right\|_{e, \mathscr{L} \mathscr{B} \rightarrow H_{\mu}^{\infty}} & \\
\leq & \frac{1}{n+1}\left\|\varphi^{\prime}\right\|_{H_{\mu}^{\infty}}\left(1+\frac{2}{1-s}\right) \frac{2}{(1-s) \ln (4 e /(1-s))} \\
& +2 \sup _{|\varphi(z)|>s} \frac{\mu(z)\left|\varphi^{\prime}(z)\right|}{(1-|\varphi(z)|) \ln (2 e /(1-|\varphi(z)|))} .
\end{aligned}
$$

Letting $n \rightarrow \infty$ and then letting $s \rightarrow 1^{-}$, we obtain that

$$
\begin{aligned}
& \left\|D C_{\varphi}\right\|_{e, \mathscr{L} \mathscr{B} \rightarrow H_{\mu}^{\infty}} \\
& \quad \leq 2 \limsup _{|\varphi(z)| \rightarrow 1^{-}} \frac{\mu(z)\left|\varphi^{\prime}(z)\right|}{(1-|\varphi(z)|) \ln (2 e /(1-|\varphi(z)|))} .
\end{aligned}
$$

The proof of the theorem is finished.

Corollary 7. Let $\varphi$ be an analytic self-map of $\mathbb{D}, \mu$ be a weight on $\mathbb{D}$, and $D_{\varphi}$ be a bounded operator from $\mathscr{L} \mathscr{B}\left(\right.$ or $\left.\mathscr{L} \mathscr{B}_{0}\right)$ to $H_{\mu}^{\infty}$. Then $D_{\varphi}$ is a compact operator from $\mathscr{L} \mathscr{B}\left(\right.$ or $\left.\mathscr{L} \mathscr{B}_{0}\right)$ to $H_{\mu}^{\infty}$ if and only if

$$
\limsup _{|\varphi(z)| \rightarrow 1^{-}} \frac{\mu(z)\left|\varphi^{\prime}(z)\right|}{(1-|\varphi(z)|) \ln (2 e /(1-|\varphi(z)|))}=0 .
$$

\section{Conflict of Interests}

The author declares that there is no conflict of interests regarding the publication of this paper.

\section{Acknowledgments}

The author thanks the referee for the careful reading of the paper and for the helpful comments and suggestions. The work is partially supported by Special Fund of Colleges and Universities in Fujian Province (no. JK2012010) and Natural Science Foundation of Fujian Province (no. 2009J01004), China.

\section{References}

[1] R. Yoneda, "The composition operators on weighted Bloch space," Archiv der Mathematik, vol. 78, no. 4, pp. 310-317, 2002.

[2] S. Stević, "On a new operator from the logarithmic Bloch space to the Bloch-type space on the unit ball," Applied Mathematics and Computation, vol. 206, no. 1, pp. 313-320, 2008.

[3] S. Ye, "Multipliers and cyclic vectors on weighted Bloch space," Mathematical Journal of Okayama University, vol. 48, pp. 135143, 2006.

[4] S. Ye, "A weighted composition operator between different weighted Bloch-type spaces," Acta Mathematica Sinica, vol. 50, pp. 927-942, 2007.

[5] S. Ye, "Weighted composition operators from $F(p, q, s)$ into logarithmic bloch space," Journal of the Korean Mathematical Society, vol. 45, no. 4, pp. 977-991, 2008.

[6] S. Ye, "Weighted composition operator between the little $\alpha$ Bloch spaces and the logarithmic Bloch," Journal of Computational Analysis and Applications, vol. 11, no. 2, pp. 443-450, 2009.

[7] S. Ye, "A weighted composition operator on the logarithmic bloch space," Bulletin of the Korean Mathematical Society, vol. 47, no. 3, pp. 527-540, 2010.

[8] S. Ye, "Products of Volterra-type operators and composition operators on logarithmic Bloch space," WSEAS Transactions on Mathematics, vol. 12, no. 2, pp. 180-188, 2013.

[9] R. A. Hibschweiler and N. Portnoy, "Composition followed by differentiation between bergman and hardy spaces," Rocky 
Mountain Journal of Mathematics, vol. 35, no. 3, pp. 843-855, 2005.

[10] S. Stević, "Norm and essential norm of composition followed by differentiation from $\alpha$-Bloch spaces to $H_{\mu}^{\infty}$," Applied Mathematics and Computation, vol. 207, pp. 225-229, 2009.

[11] H. Jarchow and J. Xiao, "Composition operators between Nevanlinna classes and Bergman spaces with weights," The Journal of Operator Theory, vol. 46, pp. 605-618, 2001.

[12] K. Madigan and A. Matheson, "Compact composition operators on the Bloch space," Transactions of the American Mathematical Society, vol. 347, pp. 2679-2687, 1995.

[13] W. Smith, "Composition operators between Bergman and Hardy spaces," Transactions of the American Mathematical Society, vol. 348, no. 6, pp. 2331-2348, 1996.

[14] J. Xiao, "Compact compositon operators on the areaNevanlinna class," Expositiones Mathematicae, vol. 17, pp. 255-264, 1999.

[15] S. Ye and Q. Hu, "Weighted composition operators on the Zygmund space," Abstract and Applied Analysis, vol. 2012, Article ID 462482, 18 pages, 2012.

[16] S. Ye and Z. Zhuo, "Weighted composition operators from Hardy to Zygmund type spaces," Abstract and Applied Analysis, vol. 2013, Article ID 365286, 10 pages, 2013.

[17] C. Zeljko and R. H. Zhao, "Weighted composition operators between different weighted Bergman spaces and different Hardy spaces," Illinois Journal of Mathematics, vol. 51, no. 2, pp. 479-498, 2007.

[18] C. Zeljko and R. H. Zhao, "Essential norm estimates of weighted composition operators between Bergman spaces on strongly pseudoconvex domains," Mathematical Proceedings of the Cambridge Philosophical Society, vol. 142, no. 3, pp. 525-533, 2007.

[19] S. Stević, "Norm of weighted composition operators from Bloch space to $H_{\mu}^{\infty}$ on the unit ball," Ars Combinatoria, vol. 88, pp. 125-127, 2008.

[20] J. H. Shapiro, "The essential norm of a composition operator," Annals of Mathematics, vol. 125, pp. 375-404, 1987.

[21] R. Donaway, Norm and essential norm estimates of compositions on Besov type spaces [M.S. thesis], University of Virgina, 1999. 


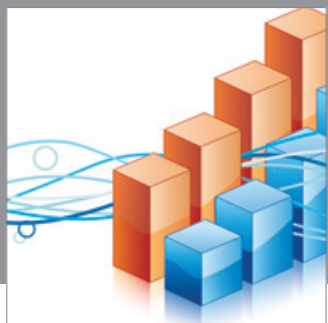

Advances in

Operations Research

mansans

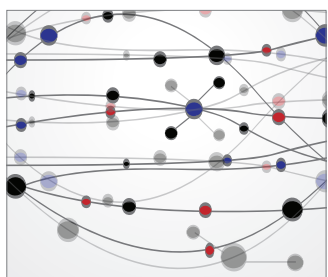

The Scientific World Journal
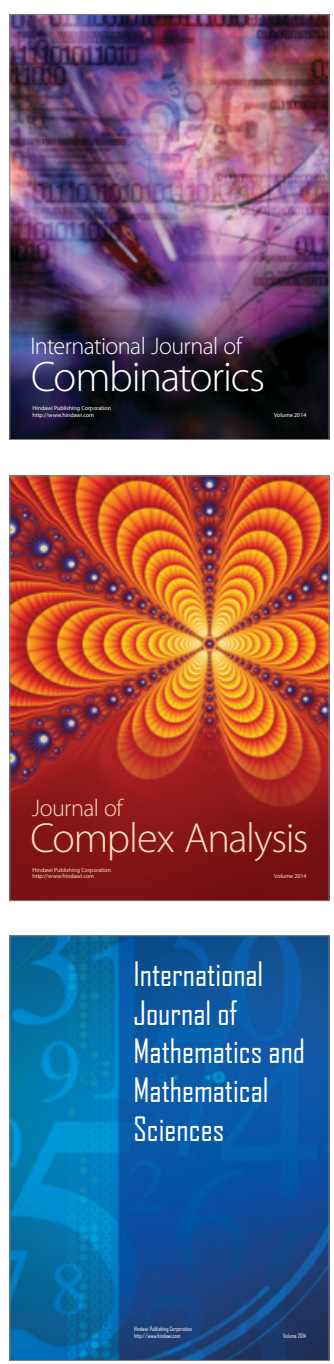
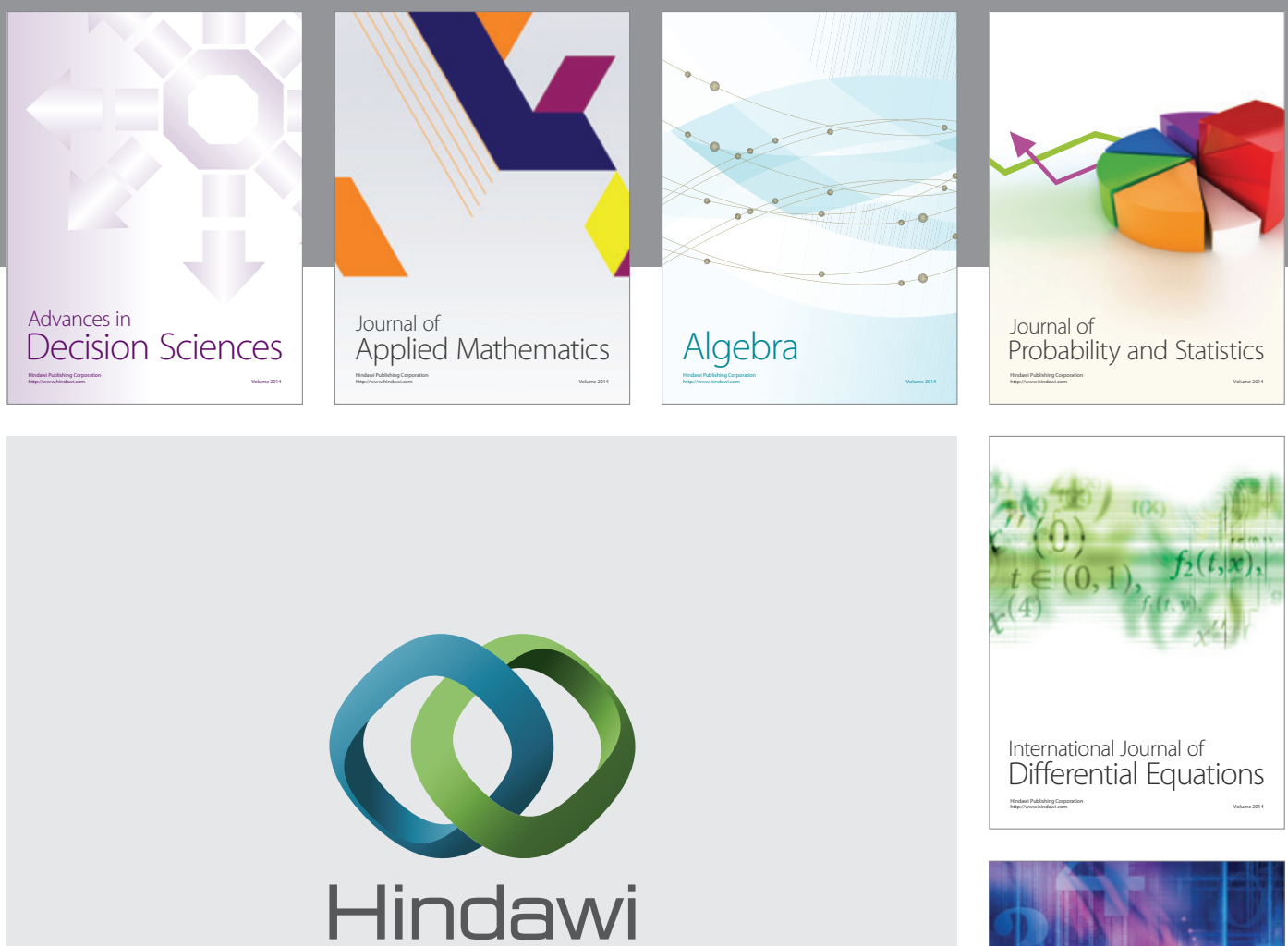

Submit your manuscripts at http://www.hindawi.com
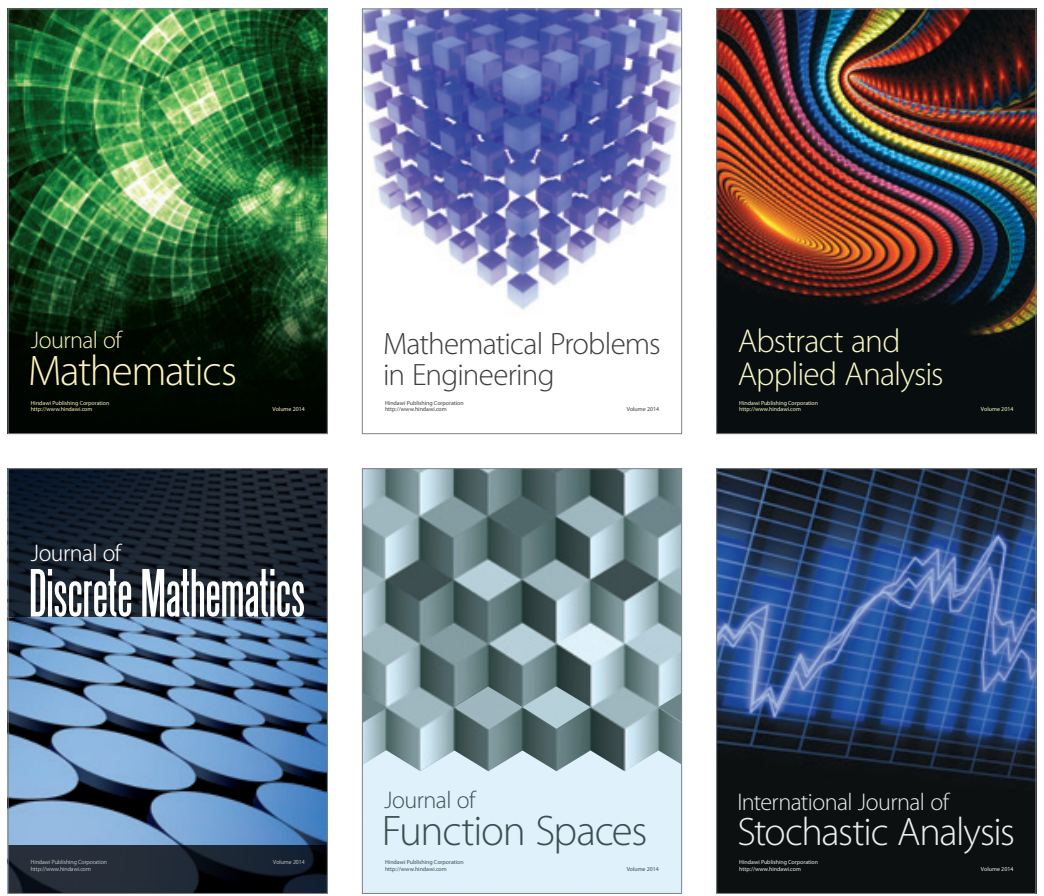

Journal of

Function Spaces

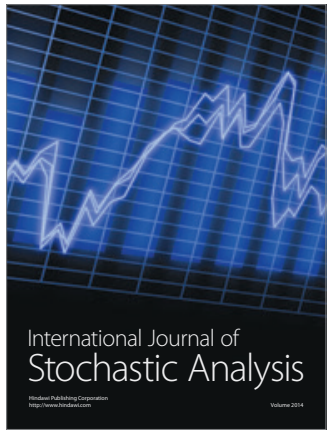

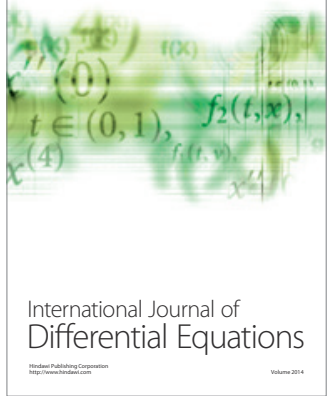
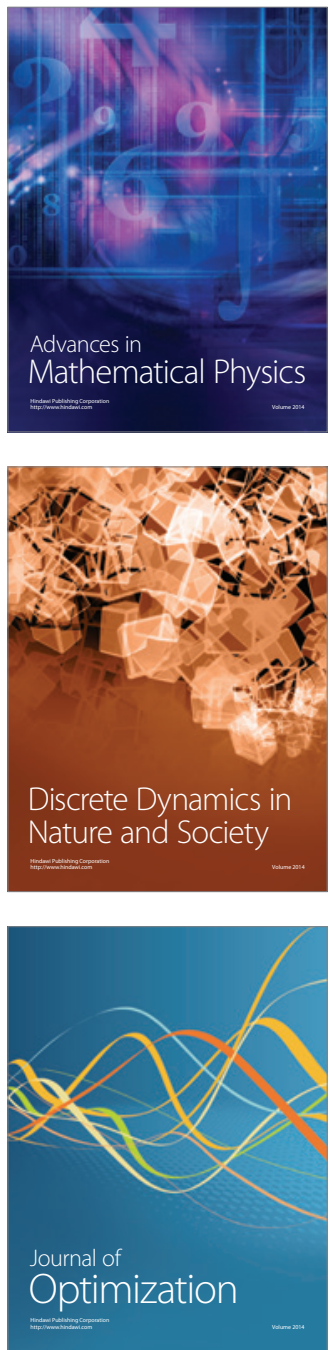